\title{
Considerations About Equations for Steady State Flow in Natural Gas Pipelines
}

In this work a discussion on the particularities of the pressure drop equations being used in the design of natural gas pipelines will be carried out. Several versions are presented according to the different flow regimes under consideration and through the presentation of these equations the basic physical support for each one is discussed as well as their feasibility.

Keywords: natural gas flow, pressure drop, gas pipelines, Renouard

Carlos Pinho

Member, ABCM

ctp@fe.up.pt

University of Porto

Rua Dr. Roberto Frias, $s / n$

4200-465 Porto, Portugal

\section{Introduction}

The design of gas pipelines and networks is commonly presented through a series of numerical procedures and recommendations, and usually flow equations are recommended by the several authors according to common design and calculation practice, without a deep analysis of the basic physical reasoning that is behind each one of such equations. In this work a discussion on the particularities of the pressure drop equations being used in the design of gas pipelines will be carried out and several versions presented. The development of the flow equation is commonly found in several books and publications in Fluid Mechanics or connected to industrial gas utilization technologies (Pritchard et al., 1978; Katz and Lee, 1990), consequently it is useless to present once again such derivation. The reader can consult the work of Mohitpour et al. (2000) where such analysis is presented for steady and unsteady state compressible fluid flow.

\section{Nomenclature}

$C, C^{\prime}=$ generic constant

$d=$ gas relative density, dimensionless

$D=$ internal diameter of pipe, $\mathrm{m}$

$E=$ potential energy term, Eq.(34), $\mathrm{Pa}^{2}$

$f=$ Darcy friction coefficient, dimensionless

$g=$ gravitational acceleration, $\mathrm{m} / \mathrm{s}^{2}$

$H=$ height of points 1 and $2, \mathrm{~m}$

$K=$ constant, dimensionless

$L=$ pipe length, $\mathrm{m}$

$M=$ molecular mass, $\mathrm{kg} / \mathrm{kmol}$

$n=$ exponent for the gas flow rate (range of values between 1.74 and 2)

$P, P^{\prime}=$ absolute pressure, $\mathrm{Pa}$

$P_{1}^{\prime}=$ absolute pressure at pipe entrance, $\mathrm{Pa}$

$P_{2}=$ absolute pressure at pipe exit, $\mathrm{Pa}$

$P_{\text {avg }}=$ flow average pressure, $\mathrm{Pa}$

$P_{\text {st }}=$ standard pressure, $1.01325 \times 10^{5} \mathrm{~Pa}$

$\dot{Q}_{s t}=$ volume gas flow rate at standard conditions, $\mathrm{m}^{3} / \mathrm{s}$

$R=$ flow resistance per unit length of pipe

$\bar{R}=$ universal gas constant, $8314.41 \mathrm{~J} /(\mathrm{kmol} \mathrm{K})$

$\mathrm{Re}=$ Reynolds number of the gas flow, dimensionless

$T=$ absolute temperature, $\mathrm{K}$

$T_{\text {avg }}=$ flow average temperature, $\mathrm{K}$

Paper accepted April, 2006. Technical Editor: Clovis R. Maliska.
$T_{\text {st }}=$ standard temperature, $288.15 \mathrm{~K}$

$z_{\text {avg }}=$ gas compressibility factor, dimensionless

$z_{\mathrm{st}}=$ compressibility factor at standard conditions, $\mathrm{z}_{\mathrm{st}} \approx 1$

\section{Greek Symbols}

$\alpha=$ coefficient, dimensionless

$\beta=$ coefficient, dimensionless

$\Delta$ max $=$ maximum variation of the friction factor, dimensionless

$\Delta P=$ pressure drop, $\mathrm{Pa}$

$\varepsilon=$ wall roughness, $\mathrm{m}$

$\eta=$ efficiency factor, dimensionless

$\mu=$ gas dynamic viscosity, $\mathrm{Pa} \mathrm{s}$

$\nu=$ gas cinematic viscosity, $\mathrm{m}^{2} / \mathrm{s}$

$\rho=$ gas density, $\mathrm{kg} / \mathrm{m}^{3}$

$\tau=$ shear stress, $\mathrm{Pa}$

$\begin{array}{ll}\text { Subscripts } \\ \text { air } & \text { air } \\ \text { app } & \text { apparent } \\ \text { avg } & \text { average } \\ c g & \text { city gas } \\ \text { cr } & \text { critical } \\ \text { ent } & \text { entrance } \\ \text { st } & \text { standard } \\ \text { sta } & \text { standard for air } \\ w & \text { wall } \\ 1 & \text { relative to the generic point } 1 \\ 2 & \text { relative to the generic point } 2\end{array}$

\section{General Equation for Steady-State Flow}

Considering the momentum equation applied to a portion of pipe of length $d x$, inside which flows a compressible fluid with an average velocity $u$, for example natural gas, assuming steady state conditions where $\rho$ is the gas density, $p$ is the gas absolute static pressure, $A$ is the area of the pipe cross section $\left(\pi D^{2} / 4\right)$ and $d H$ represents a variation in high, the resultant differential equation is,

$$
u d u+\frac{d P}{\rho}+g d H+f \frac{d x}{D} \frac{u^{2}}{2}=0
$$

In the above equation $f$ is the Darcy friction coefficient which is related with the wall shear stress by means of,

$$
f=\frac{8 \tau_{w}}{\rho u^{2}}
$$


Before proceeding to the integration of Eq. (1) between two generic points 1 and 2 of the pipeline, it is most convenient to simplify the viscous dissipation term to easy up its integration, otherwise the function $u^{2}=f(x)$ should be known to carry on with the integration. But as $\rho u=\dot{m} / A=C$ is a constant, according to the steady state conditions, then,

$$
\rho^{2} u d u+\rho d P+\rho^{2} g d H+f \frac{d x}{D} \frac{C^{2}}{2}=0
$$

To integrate each one of the terms of the previous equation, a more detailed discussion will follow.

\section{Kinetic Energy Term $\left(\rho^{2} u d u\right)$}

As $\rho=C / u$ then,

$$
\int_{u_{1}}^{u_{2}} \frac{C^{2}}{u} d u=C^{2} \ln \left(\frac{u_{2}}{u_{1}}\right)
$$

\section{Pressure Force Work Term $(\rho d P)$}

$$
\text { As } \rho=\frac{P M}{z \bar{R} T}
$$

the integration of this term becomes,

$$
\int_{P_{1}}^{P_{2}} \rho d P=\int_{P_{1}}^{P_{2}} \frac{P M}{z \bar{R} T} d P=\frac{M}{z_{\text {avg }} \bar{R} T_{\text {avg }}} \int_{P_{1}}^{P_{2}} P d P=\frac{M}{z_{\text {avg }} \bar{R} T_{\text {avg }}} \frac{\left(P_{2}^{2}-P_{1}^{2}\right)}{2} \text { (5) }
$$

Some explanation must be presented about the parameters, $T_{\text {avg }}$ and $z_{\text {avg. }}$. To follow an easier approach for the integration, average values were used for the compressibility factor and gas temperature. The gas average temperature can be obtained through Mohitpour et al. (2000),

$$
T_{\text {avg }}=\frac{T_{1}+T_{2}}{2}
$$

whereas the average pressure will be determined through $(d P / d x \propto 1 / P)$,

$$
P_{a v g}=\frac{\int_{1}^{2} P d x}{\int_{1}^{2} d x}=\frac{\int_{1}^{2} P^{2} d P}{\int_{1}^{2} P d P}=\frac{2}{3}\left[P_{1}+P_{2}-\frac{P_{1} P_{2}}{P_{1}+P_{2}}\right]
$$

Finally $z_{\text {avg }}$ will be determined based on the above obtained average values for $P$ and $T$ through adequate tables or formulae, Smith (1990).

\section{Potential Energy Term $\left(\rho^{2} g d H\right)$}

$$
\int_{H_{1}}^{H_{2}} \rho^{2} g d H=\int_{H_{1}}^{H_{2}}\left(\frac{P M}{z \bar{R} T}\right)^{2} g d H
$$

It is again assumed that $(P M /(z \bar{R} T))^{2}$ can be determined by average values which greatly simplifies the integration, Mohitpour et al. (2000), and the final result is

$$
\frac{g P_{a v g}^{2} M^{2}}{z_{\text {avg }}^{2} \bar{R}^{2} T_{\text {avg }}^{2}}\left(H_{2}-H_{1}\right)
$$

This approach is justified by the fact that there is no simple mathematical relationship among height, pressure and temperature, and the introduced error is meaningless.

Energy Dissipation by Viscous Friction $\left(f \frac{d x}{D} \frac{C^{2}}{2}\right)$

$$
\int_{x_{1}}^{x_{2}} \frac{f C^{2}}{2 D} d x=f \frac{\left(x_{2}-x_{1}\right)}{D} \frac{C^{2}}{2}=f \frac{L}{D} \frac{C^{2}}{2}
$$

where $L$ is the pipe length between points 1 and 2 .

The addition of the previously determined terms yields,

$$
C^{2} \ln \left(\frac{u_{2}}{u_{1}}\right)+\frac{M}{z_{\text {avg }} \bar{R} T_{\text {avg }}} \frac{\left(P_{2}^{2}-P_{1}^{2}\right)}{2}+\frac{g P_{\text {avg }}^{2} M^{2}}{z_{\text {avg }}^{2} \bar{R}^{2} T_{\text {avg }}^{2}}\left(H_{2}-H_{1}\right)+f \frac{L}{D} \frac{C^{2}}{2}=0
$$

and as usually, the kinetic energy term is negligible when compared with the other terms,

$$
\frac{M}{z_{\text {avg }} \bar{R} T_{\text {avg }}} \frac{\left(P_{2}^{2}-P_{1}^{2}\right)}{2}+\frac{g P_{\text {avg }}^{2} M^{2}}{z_{\text {avg }}^{2} \bar{R}^{2} T_{\text {avg }}^{2}}\left(H_{2}-H_{1}\right)+f \frac{L}{D} \frac{C^{2}}{2}=0
$$

Normally the volume flow rate at standard conditions $\dot{Q}_{s t}$ $\left(288.15 \mathrm{~K}\right.$ and $\left.1.1325 \times 10^{5} \mathrm{~Pa}\right)$ is used, instead of the constant $C$, and combining the definition of this constant and of the mass flow rate

$$
C^{2}=\frac{\rho_{s t}^{2} \dot{Q}_{s t}^{2}}{A^{2}}=\frac{16 P_{s t}^{2} M^{2} \dot{Q}_{s t}^{2}}{\pi^{2} D^{4} z_{s t}^{2} \bar{R}^{2} T_{s t}^{2}}
$$

and because for a perfect gas,

$$
M=d M_{\text {air }}
$$

where $d$ is the gas density relative to the air and $M_{\text {air }}=28.9625 \approx 29$ $\mathrm{kg} / \mathrm{kmol}$, the combination of Eqs. (12) to (14) gives in SI units that,

$\dot{Q}_{s t}=\pi \sqrt{\frac{\bar{R}}{464}} \frac{z_{s t} T_{s t}}{P_{s t}}\left[\frac{\left(P_{1}^{2}-P_{2}^{2}\right)-\frac{58 d P_{\text {avg }}^{2} g\left(H_{2}-H_{1}\right)}{\bar{R} T_{\text {avg }} z_{\text {avg }}}}{L d T_{\text {avg }} z_{\text {avg }}}\right]^{1 / 2} \frac{D^{2.5}}{\sqrt{f}} \eta(15)$

According to Osiadacz (1987), the real gas flow in a pipe is inferior to that calculated by means of the flow equation, namely Eq. (15) when $\eta=1$, because of extra friction imposed by fittings like bends, tees, valves and also by other effects like corrosion, fouling and dust/rust deposition. To account for such extra flow reductions in a simple and effective way, it is a common practice to use a corrective multiplying factor, the efficiency factor $\eta$, which usually takes values between 0.8 and 1 . Mohitpour et al. (2000) suggest $\eta$ values between 0.92 and 0.97 , although experience recommends that for old piping it can be as low as 0.7, Osiadacz (1987).

Sometimes the influence of $\eta$ is introduced as a correction of the pipe length, i.e. $L / \eta^{2}$ replaces $L$ and accordingly an equivalent length is used which can take values between $1.56 L$ and $L$, corresponding to the $0.8<\eta<1$ range. 
Considering that $\rho_{s t}=M P_{s t} /\left(z_{s t} \bar{R} T_{s t}\right), \quad$ and that $\rho_{\text {avg }}=M P_{\text {avg }} /\left(z_{\text {avg }} \bar{R} T_{\text {avg }}\right)$, while excluding for the sake of simplicity the factor $\eta$, a simpler although less common form can be given to the last equation,

$\dot{Q}_{s t}=\frac{\pi}{4 \rho_{s t}}\left(\frac{29 d}{z_{\text {avg }} \bar{R} T_{\text {avg }} L}\left[\left(P_{1}^{2}-P_{2}^{2}\right)-2 P_{a v g} \rho_{\text {avg }} g\left(H_{2}-H_{1}\right)\right]\right)^{1 / 2} \frac{D^{2.5}}{\sqrt{f}}(16)$

Replacing all the constants of the above equation that are outside the square brackets by $C^{\prime}$,

$$
\dot{Q}_{s t}=C^{\prime}\left[\frac{\left(P_{1}^{2}-P_{2}^{2}\right)-2 P_{a v g} \rho_{a v g} g\left(H_{2}-H_{1}\right)}{L d T_{\text {avg }} z_{\text {avg }}}\right]^{1 / 2} \frac{1}{\sqrt{f}} D^{2.5}
$$

For a horizontal pipe and whenever $\left.\left.\left(P_{1}^{2}-P_{2}^{2}\right)\right\rangle\right\rangle 2 P_{\text {avg }} \rho_{\text {avg }} g\left(H_{2}-H_{1}\right)$ the potential energy or elevation term can be neglected and consequently

$$
\dot{Q}_{s t}=C^{\prime}\left[\frac{\left(P_{1}^{2}-P_{2}^{2}\right)}{L d T_{a v g} z_{\text {avg }}}\right]^{1 / 2} \frac{1}{\sqrt{f}} D^{2.5}
$$

With this last equation, the importance of the ratio $1 / \sqrt{f}$, known as the transmission factor, as well of the pipe internal diameter $D$, on the gas flow rate, is easily evaluated. The transmission factor is an important parameter because it is a simple representation of the gas transmissivity inside the duct, Smith et al. (1956). On the other end, the pipe internal diameter is another important parameter for the design of pipeline systems as, for example, in the case of a duplication of its value the flow will increase $2^{2.5} \approx 5.66$ times.

Reworking the last equation, the gas standard volume flow can be written as,

$$
\dot{Q}_{s t}^{2}=C^{2} \frac{\left(P_{1}^{2}-P_{2}^{2}\right)}{L d T_{a v g} z_{\text {avg }}} \frac{1}{f} D^{5}
$$

or in fact,

$$
P_{1}^{2}-P_{2}^{2}=\dot{Q}_{s t}^{2} \frac{1}{C^{\prime 2}}\left(L d T_{\text {avg }} z_{\text {avg }}\right) \frac{f}{D^{5}}
$$

and in a generic way it can be said that,

$$
P_{1}^{2}-P_{2}^{2}=R L \dot{Q}_{s t}^{n}
$$

where $n$ e $R$ are dependent upon the equation being used for the calculation of the transmission factor, $1 / \sqrt{f}$.

\section{Flow Regimes}

Before discussing the equations available in the literature for the calculation of the friction factor or, which is the same, for the calculation of the transmission factor, it is more convenient to consider, even in a broad sense, the different flow regimes usually found in pipeline gas transportation. For typical transmission lines with high pressure gas and moderate to high gas flow rates, one of the two following situations is usually observed: Fully turbulent flow (rough pipe flow) or partially turbulent flow (smooth pipe flow).

Considering the definition of the Reynolds number, the relation between the gas average velocity and its volume flow rate, and knowing that $\rho \dot{Q}=\rho_{s t} \dot{Q}_{s t}=\dot{m}$ for steady state conditions

$$
\operatorname{Re}=\frac{4 \rho_{s t} \dot{Q}_{s t}}{\mu \pi D}
$$

As, $\rho_{s t}=P_{s t} M /\left(z_{s t} \bar{R} T_{s t}\right)$ where $z_{s t} \approx 1$ and $M \approx 29 d$

$$
\operatorname{Re}=\frac{4 \dot{Q}_{s t} 29 d P_{s t}}{\mu \pi D \bar{R} T_{s t}}
$$

But $\bar{R}=8314.41 \mathrm{~J} /(\mathrm{kmol} \mathrm{K})$ and remembering that standard conditions are defined as $P_{s t}=1.01325 \times 10^{5} \mathrm{~Pa}$ and $T_{s t}=288.15 \mathrm{~K}$ then,

$$
\operatorname{Re}=1.5616 \frac{\dot{Q}_{s t} d}{\mu D}
$$

Assuming a gas dynamic viscosity of $1.0758 \times 10^{-5} \mathrm{~Pa}$ s typical of natural gases, Mohitpour et al. (2000), the previous equation can be further simplified,

$$
\operatorname{Re}=145158.7 \frac{\dot{Q}_{s t} d}{D}
$$

As known, for Re smaller than 2100 the flow is laminar, whereas for Re above 2100 the flow is considered turbulent. Between laminar and turbulent flows there is a transition region, for which there are no available pressure drop correlations.

\section{Laminar Flow Regime}

Although usually gas flow situations in pipelines are turbulent, for the sake of completeness of analysis, the laminar flow situation is also covered in the present text.

When the laminar flow is completely developed through a duct of circular cross section, the Darcy friction factor, independent of pipe roughness, is given by White (1999),

$$
f \mathrm{Re}=64
$$

According to Shah and London (1978), the laminar flow inside a duct is fully developed beyond a certain entrance length $L_{\text {ent }}$, determined by,

$$
\frac{L_{e n t}}{D}=0.59+0.056 \mathrm{Re}
$$

For a region when the flow is still under development, i.e. for lengths shorter than the entrance length, the average or apparent Darcy friction factor can be calculated through Shah and London (1978)

$$
f_{a p p} \operatorname{Re}=\frac{13.76}{\left(x^{+}\right)^{1 / 2}}+\frac{5 /\left(4 x^{+}\right)+64-13.76 /\left(x^{+}\right)^{1 / 2}}{1+0.00021\left(x^{+}\right)^{-2}}
$$

where the dimensionless length is given by $x^{+}$ 


$$
x^{+}=\frac{x}{D \operatorname{Re}}
$$

$x$ is the pipe length and $D$ is the internal diameter of pipe. When $x^{+}$ $\rightarrow \infty$, then $f_{\text {app }} \operatorname{Re} \rightarrow 64$.

\section{Partial and Fully Turbulent Flow Regions}

In the partially turbulent flow the laminar sublayer thickness is bigger than the pipe wall absolute roughness, and there is then a laminar region covering the tube inner wall and a turbulent region outside it. It is as if there was a turbulent flow inside a smooth walled pipe and that is the reason why the pipe wall is designated as hydraulically smooth, the pressure drop is found to be independent of the roughness of the pipe, Munson et al. (1998). The Darcy friction factor in this situation can be calculated by the Prandtl-Von Karman equation, Mohitpour et al. (2000),

$$
\frac{1}{\sqrt{f}}=-2 \log _{10}\left(\frac{2.825}{\operatorname{Re} \sqrt{f}}\right)
$$

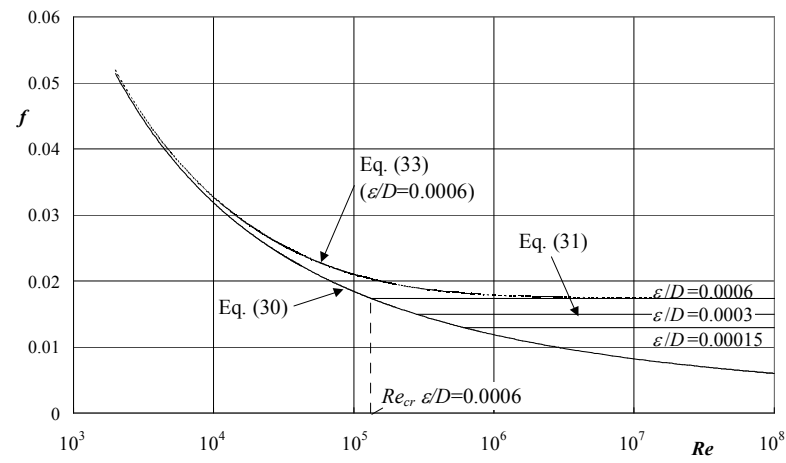

Figure 1. Influence of the relative roughness in the regime transition from hydraulically smooth to rough pipes turbulent flow.

With the increase of the Reynolds number the laminar sublayer thickness decreases, the pipe roughness gets increasingly important, disrupting the laminar sublayer, and after a brief transition region, the friction coefficient becomes independent of the Reynolds number, Munson et al. (1998), i.e. these are the conditions of the fully turbulent regime. In such circumstances this Darcy factor is determined by the Nikuradse equation, Mohitpour et al. (2000),

$$
\frac{1}{\sqrt{f}}=-2 \log _{10}\left(\frac{\varepsilon / D}{3.7}\right)
$$

Equation (30) for smooth pipes can be used until the influence of the viscous sublayer is replaced by the influence of pipe roughness Smith (1990) and from then on Eq. (31) must be used. As can be seen in Fig. 1 the transition Reynolds is dependent upon the relative roughness and the smaller this is, the later is the transition region.

Designating by critical Reynolds number, the Reynolds at which value there is an abrupt change from turbulent flow in smooth pipes towards turbulent flow in rough pipes, or fully turbulent flow $\mathrm{Re}_{\mathrm{cr}}$, a very convenient graphic can be plotted, Fig. 2, which presents through a continuous line the relationship between $\operatorname{Re}_{\text {cr }}$ and $\varepsilon / D$ or in other words, it shows the border between these two flow regimes. Such line can be fitted by the following equation,

$$
\operatorname{Re}_{\text {cr }}=35.235(\varepsilon / D)^{-1.1039}
$$

Many researchers, as referred by Smith (1990), adopt a modification of the Colebrook-White equation, using the 2.825 constant instead of 2.51 , valid for the partial turbulent, transition and fully turbulent regions,

$$
\frac{1}{\sqrt{f}}=-2 \log _{10}\left(\frac{\varepsilon / D}{3.7}+\frac{2.825}{\operatorname{Re} \sqrt{f}}\right)
$$

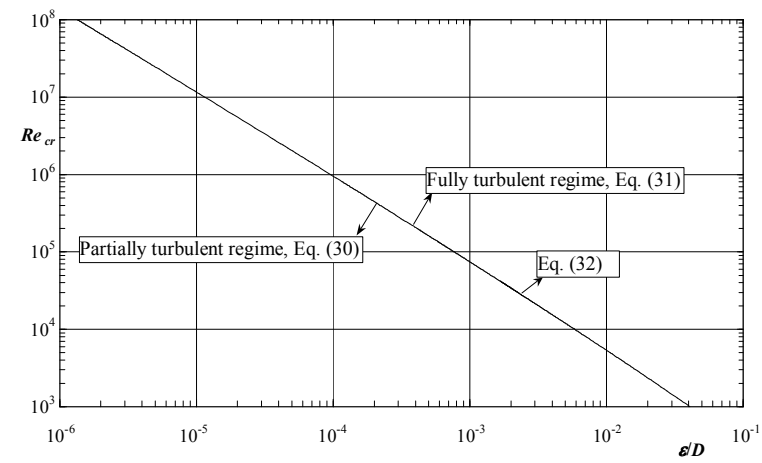

Figure 2. The critical Reynolds number $\mathrm{Re}_{c r}$ and the relative roughness.

However, this same author (Smith, 1990), refers that experimental data for commercial pipes do not follow this modified Colebrook-White equation, rather have an abrupt change between partial and fully turbulent regimes and consequently Eqs. (30) and (31) should be used instead of Eq. (33). In such circumstances, Eq. (32) is crucial for defining the correct choice. Figure 1 is an optimum guidance for a clear comprehension of the situation.

\section{Most Frequent Flow Regimes}

The gas velocity inside pipelines is limited either to reduce erosion or noise and consequently the Reynolds becomes also limited. It is then interesting to compare this practical limit value with the critical Reynolds previously defined for inter-turbulent regime transition. Table 1 shows a comparison between these two Reynolds numbers. Flow conditions used for calculations were, absolute pressure of 5 atm to give a maximum $\mathrm{Re}$ limit for distribution and utilization networks; natural gas density 0.65 also for the same reason as $\rho=P 29 d /(\bar{R} T)$; temperature $288.15 \mathrm{~K}$; average gas velocity $10 \mathrm{~m} / \mathrm{s}$, which is for the great majority of situations a limit design value; gas absolute viscosity $1.0758 \times 10^{-5}$ Pa s, pipe absolute roughness $0.0191 \mathrm{~mm}$ Smith et al. (1956), which is a lower limit for some steel pipes and a good reference absolute maximum value for all copper and polyethylene pipe tubes, value that imposes a maximum critical Reynolds for steel pipes and a minimum for copper and polyethylene pipe which is likely to appear in normal working conditions, Eq. (32).

Table 1. Comparison between $\mathbf{R e}_{\mathrm{cr}}$ and $\mathbf{R e}_{\text {lim }}$

\begin{tabular}{|c|c|c|c|}
\hline $\begin{array}{c}\text { Nominal } \\
\text { diameter }\end{array}$ & $\mathrm{Re}_{\lim }$ & $\begin{array}{c}\mathrm{Re}_{\mathrm{cr}} \\
(\varepsilon=0.0191 \mathrm{~mm})\end{array}$ & $\begin{array}{c}\mathrm{Re}_{\mathrm{cr}} \\
(\varepsilon=0.046 \mathrm{~mm})\end{array}$ \\
\hline $1 / 2^{\prime \prime}$ & 45480 & 46029 & 18813 \\
\hline $3 / 4^{\prime \prime}$ & 68221 & 72014 & 26818 \\
\hline $1^{\prime \prime}$ & 90961 & 98932 & 42575 \\
\hline $2^{\prime \prime}$ & 181922 & 212640 & 87089 \\
\hline $4^{\prime \prime}$ & 363844 & 457037 & 183586 \\
\hline $6^{\prime \prime}$ & 545765 & 715054 & 284565 \\
\hline $8^{\prime \prime}$ & 727687 & 982333 & 380583 \\
\hline $10^{\prime \prime}$ & 909609 & 1256717 & 489533 \\
\hline
\end{tabular}


As can be seen from the observation of Table 1 the limit Reynolds is always inferior to the critical Reynolds which means that in distribution and utilization networks, the flow subregime is partially turbulent, i.e. hydraulically smooth pipes. Fully turbulent flow will occur mainly in the first and second level networks where pressures are higher, imposing for the same maximum velocity, an increase of the specific volume and consequently of the Reynolds number.

The previous case is substantially changed if an absolute roughness of $0.046 \mathrm{~mm}$ is used, which is the typical suggested value for commercial steel pipes (White, 1999) (with an uncertainty in its definition of $\pm 30 \%$ ). In these circumstances fully turbulent flow can also occur for all available steel pipe diameters, see Table 1, in practical terms only copper or polyethylene pipes can guarantee exclusive conditions for partially turbulent flow.

\section{Most Used Equations for Steady Flow}

Equation (15) can take a simpler form when constants are replaced by their corresponding values and the potential energy term is replaced by

$$
E=0.06843 d\left(H_{2}-H_{1}\right) \frac{P_{a v g}^{2}}{T_{a v g} z_{\text {avg }}}
$$

so that,

$$
\dot{Q}_{s t}=13.2986 \frac{T_{s t}}{P_{s t}}\left[\frac{\left(P_{1}^{2}-P_{2}^{2}\right)-E}{L d T_{a v g} z_{\text {avg }}}\right]^{1 / 2} \frac{D^{2.5}}{\sqrt{f}}
$$

For low pressure flows, i.e., when the gauge pressure is lower than 50 mbar, pressure difference $P_{1}^{2}-P_{2}^{2}$ can be simplified, Osiadacz (1987),

$$
P_{1}^{2}-P_{2}^{2}=\left(P_{1}-P_{2}\right) 2\left(P_{1}+P_{2}\right) / 2
$$

and taking into consideration that for low pressures,

$$
\left(P_{1}+P_{2}\right) / 2=P_{a v g}^{\prime}
$$

where $P_{a v g}^{\prime}$ is the average pressure inside the conveying duct,

$$
P_{1}^{2}-P_{2}^{2}=\left(P_{1}-P_{2}\right) \cdot 2 P_{\text {avg }}^{\prime}
$$

The result is,

$$
\dot{Q}_{s t}=13.2986 \frac{T_{s t}}{P_{s t}}\left[\frac{\left(P_{1}-P_{2}\right) 2 P_{a v g}^{\prime}-E}{L d T_{a v g} z_{\text {avg }}}\right]^{1 / 2} \frac{D^{2.5}}{\sqrt{f}}
$$

In the above equation, $P_{1}$ and $P_{2}$ can be either in absolute or gauge pressures. As shown, low pressure equations are but simplifications of high pressure equations, which have been mathematically reworked in order to ease up calculations. High pressure equations have universal application instead of low pressure equations, which have a narrower field of application.

As the equation for the transmission factor is generically,

$$
\frac{1}{\sqrt{f}}=\alpha \operatorname{Re}^{\beta}
$$

while the Reynolds number can be written as,

$$
\operatorname{Re}=\frac{4 \dot{Q}_{s t} 29 d P_{s t}}{\mu \pi D \bar{R} T_{s t}}
$$

Equation (15) can reworked to take a more general form

$$
\begin{aligned}
& \dot{Q}_{s t}=\alpha^{\frac{1}{(1-\beta)}} \pi\left(\frac{\bar{R}}{116}\right)^{\frac{(0.5-\beta)}{(1-\beta)}} \\
& \frac{1}{2^{\frac{1}{(1-\beta)}}} \frac{1}{\mu^{\frac{\beta}{(1-\beta)}}} \frac{T_{s t}}{P_{s t}}\left[\frac{\left(P_{1}^{2}-P_{2}^{2}\right)-E}{L d^{(1-2 \beta)} T_{\text {avg }} z_{\text {avg }}}\right]^{\frac{0.5}{(1-\beta)}} D^{\frac{(2.5-\beta)}{(1-\beta)}}
\end{aligned}
$$

In the next sections most common equations used for the calculation of pressure drops in gas pipelines are discussed in the light of what has previously been said. All equations have been converted to SI units although several of them are known in quite different systems of units.

\section{Equations for Turbulent Flows in Hydraulically Smooth Pipes}

\section{Panhandle A Equation}

In this case the transmission factor is given by,

$$
\frac{1}{\sqrt{f}}=3.436 \operatorname{Re}^{0.07305}
$$

which means that $\alpha=3.436$ and $\beta=0.07305$. Reworking Eq. (41) with these values, the Panhandle A equation in S.I. units is obtained

$$
\dot{Q}_{s t}=40.2970 \frac{1}{\mu^{0.0788}} \frac{T_{s t}}{P_{s t}}\left[\frac{\left(P_{1}^{2}-P_{2}^{2}\right)-E}{L d^{0.8539} T_{\text {avg }} z_{\text {avg }}}\right]^{0.5394} D^{2.6182}
$$

According to the suggestion frequently found in the technical literature, Mohitpour et al. (2000), that for this equation, $\mu=$ $1.0415 \times 10^{-5} \mathrm{~Pa} \mathrm{~s}$, the previous equation becomes,

$$
\dot{Q}_{s t}=99.5211 \frac{T_{s t}}{P_{s t}}\left[\frac{\left(P_{1}^{2}-P_{2}^{2}\right)-E}{L d^{0.8539} T_{\text {avg }} z_{\text {avg }}}\right]^{0.5394} D^{2.6182}
$$

\section{AGA Partially Turbulent Equation}

The American Gas Association equation for partially turbulent uses the transmission factor given by the Prandtl-von Karman expression,

$$
\frac{1}{\sqrt{f}}=-2 \log _{10}\left(\frac{2.825}{\operatorname{Re} \sqrt{f}}\right)
$$

and replacing it into Eq. (41) gives,

$\dot{Q}_{s t}=13.2986 \frac{T_{s t}}{P_{s t}}\left[\frac{\left(P_{1}^{2}-P_{2}^{2}\right)-E}{L d T_{\text {avg }} z_{\text {avg }}}\right]^{0.5}\left[-2 \log _{10}\left(\frac{2.825}{\operatorname{Re} \sqrt{f}}\right)\right] D^{2.5}$ 


\section{White}

To simplify the calculation of the transmission factor and assuring at the same time that the resulting friction factors are close to those obtained from a reference equation like that of Prandtl-von Karman, a good approach is to use the White equation (White, 1979) for $f$. This equation besides having a small error band between $+0.24 \%$ and $-3.23 \%$, for the calculated results, when the Reynolds goes from 2000 to $2 \times 10^{6}$, has also the advantage of the explicitness. It must be stressed that, among the several equations presented by Branco et al. (2001) the White equation, shown next, is the one that presents smaller deviations when compared with the Prandtl-von Karman (or AGA partially turbulent) equation,

$$
\frac{1}{\sqrt{f}}=\frac{1}{\sqrt{1.02}}\left(\log _{10} \mathrm{Re}\right)^{1.25}
$$

The corresponding flow equation is obtained through the introduction of the White Eq. (46) into Eq. (41) giving

$$
\dot{Q}_{s t}=13.2986 \frac{T_{s t}}{P_{s t}}\left[\frac{\left(P_{1}^{2}-P_{2}^{2}\right)-E}{L d T_{\text {méd. }} z_{\text {méd. }}}\right]^{0.5}\left[\frac{1}{\sqrt{1.02}}\left(\log _{10} \operatorname{Re}\right)^{1.25}\right] D^{2.5}
$$

Figure 3 is a comparison of friction coefficients used in the Panhandle A, AGA partially turbulent and White equations. As can be seen the $f$ corresponding to the last equation has higher values than those for Panhandle A at low Reynolds numbers. This fact is a demonstration of how limited is the range of application of several of the available equations as many of them were developed for particular situations, Schroeder (2001). As previously referred it is evident the good superimposition of friction factor values obtained with the AGA partially turbulent (or Prandtl-von Karman) and the White equations.

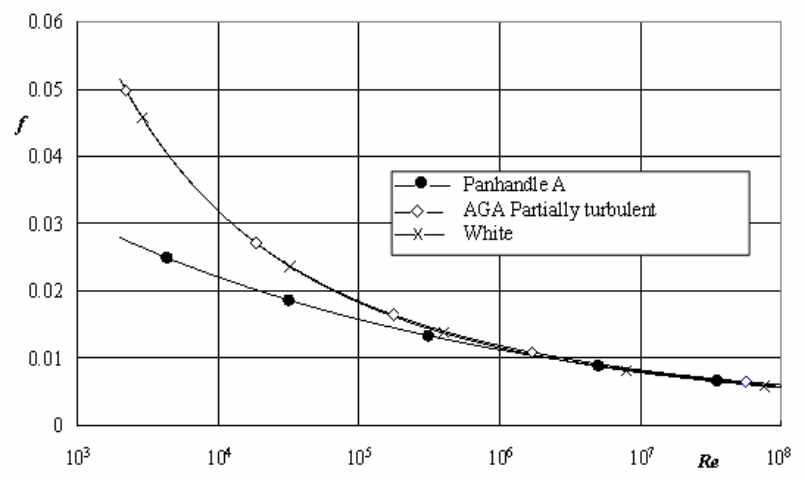

Figure 3 Comparison of attrition factors for Panhandle A, AGA p/t and White equations.

\section{Turbulent Flow in Rough Pipes}

\section{Panhandle B Equation}

The transmission factor for this equation is given by,

$$
\frac{1}{\sqrt{f}}=8.245 \operatorname{Re}^{0.01961}
$$

and so the Panhandle $B$ equation is obtained by introducing into Eq. (41), $\alpha=8.245$ and $\beta=0.01961$,

$$
\dot{Q}_{s t}=108.080 \frac{1}{\mu^{0.020}} \frac{T_{s t}}{P_{s t}}\left[\frac{\left(P_{1}^{2}-P_{2}^{2}\right)-E}{L d^{0.9608} T_{\text {avg }} z_{\text {avg }}}\right]^{0.510} D^{2.53}
$$

Using the typical viscosity of $\mu=1.0758 \times 10^{-5} \mathrm{~Pa} \mathrm{~s}$, the previous equation becomes,

$$
\dot{Q}_{s t}=135.8699 \frac{T_{s t}}{P_{s t}}\left[\frac{\left(P_{1}^{2}-P_{2}^{2}\right)-E}{L d^{0.9608} T_{\text {avg }} z_{\text {avg }}}\right]^{0.510} D^{2.53}
$$

\section{Weymouth Equation}

According to Mohitpour et al. (2002) this equation overestimates the pressure drop calculation and because of that it is most frequently used in the design of distribution networks in spite of being less exact than other equations. In this case the transmission factor is given by,

$$
\frac{1}{\sqrt{f}}=10.3196 D^{\frac{1}{6}}
$$

with the pipe inner diameter in meters.

Replacing this transmission factor into Eq. (41) the result is,

$$
\dot{Q}_{s t}=137.2364 \frac{T_{s t}}{P_{s t}}\left[\frac{\left(P_{1}^{2}-P_{2}^{2}\right)-E}{L d T_{\text {avg }} z_{\text {avg }}}\right]^{1 / 2} D^{8 / 3}
$$

\section{AGA Fully Turbulent Equation}

This is one of the most recommended and used equations for this type of flow, being able to estimate with high precision flow and pressure drop values, if pipe roughness is known with correctness. The transmission factor is given by the Nikuradse expression, Eq. (31),

$$
\frac{1}{\sqrt{f}}=-2 \log _{10}\left(\frac{\varepsilon / D}{3.7}\right)
$$

which after being replaced into Eq. (41) gives,

$$
\dot{Q}_{s t}=13.2986 \frac{T_{s t}}{P_{s t}}\left[\frac{\left(P_{1}^{2}-P_{2}^{2}\right)-E}{L d T_{\text {avg }} z_{\text {acg }}}\right]^{0.5}\left[-2 \log _{10}\left(\frac{\varepsilon / D}{3.7}\right)\right] D^{2.5}
$$

\section{Modified Colebrook-White Equation}

This equation combines the three flow regimes, partially turbulent, transition and fully turbulent and it is recommended (Mohitpour et al., 2002) when the system is operating in the transition region between both regimes, although other authors have different opinions about this subject (Smith, 1990; Gersten et al., 2000). The transmission factor is given by a modification of the Colebrook-White equation where the constant 2.51 was replaced by $2.825(18)$ to achieve better agreement with experimental data at higher Reynolds numbers (Gersten et al., 2000 ).

$$
\frac{1}{\sqrt{f}}=-2 \log _{10}\left(\frac{\varepsilon / D}{3.7}+\frac{2.825}{\operatorname{Re} \sqrt{f}}\right)
$$


and consequently the Modified Colebrook-White flow equation will be,

$$
\dot{Q}_{s t}=13.2986 \frac{T_{s t}}{P_{s t}}\left[\frac{\left(P_{1}^{2}-P_{2}^{2}\right)-E}{L d T_{\text {avg }} z_{\text {avg }}}\right]^{0.5}\left[-2 \log _{10}\left(\frac{\varepsilon / D}{3.7}+\frac{2.825}{\operatorname{Re} \sqrt{f}}\right)\right] D^{2.5}
$$

\section{Gersten et al. Equation}

This equation is the recent result of the work by GERG (Groupe Europeen de Recherches Gazières) founded in 1961 and composed by members from eight european countries.

It is an equation valid for both regimes, similarly to what happened with the previously considered Modified ColebrookWhite equation, but according to its proponents this equation reproduces with high fidelity the transition between partially and fully turbulent flow as shown by the experimental results, i.e. an abrupt change on the transmission factor with the Reynolds number.

The transmission factor is then given by Gersten et al. (2000),

$$
\frac{1}{\sqrt{f}}=-\frac{2}{n} \log _{10}\left[\left(\frac{\varepsilon / D}{3.71}\right)^{n}+\left(\frac{1.499}{t \operatorname{Re} \sqrt{f}}\right)^{0.942 \cdot n t}\right]
$$

while the corresponding flow equation is

$$
\begin{aligned}
& \dot{Q}_{s t}=13.2986 \frac{T_{s t}}{P_{s t}}\left[\frac{\left(P_{1}^{2}-P_{2}^{2}\right)-E}{L d T_{\text {avg }} z_{\text {avg }}}\right]^{0.5} \\
& \left\{-\frac{2}{n} \log _{10}\left[\left(\frac{\varepsilon / D}{3.71}\right)^{n}+\left(\frac{1.499}{t \operatorname{Re} \sqrt{f}}\right)^{0.942 n \cdot t}\right]\right\} D^{2.5}
\end{aligned}
$$

The parameter $t$ is equivalent to the efficiency factor $\eta$ present in the Eq. (22) and similarly, it is equal to the unity in the absence of localized pressure drops. Parameter $n$ has no importance for hydraulically smooth pipe flows as well as fully rough flow, but on the contrary it is fundamental for the transition region between these two flows.

For $n=1$ there is a smooth transition, as suggested by the Modified Colebrook-White equation, but on the other end, the abrupt transition as shown in the experimental natural gas data, is well described for $n=10$.

\section{Comparison of Friction Factors}

Figure 4 has a comparison among several friction factors used in the above mentioned equations for fully turbulent flow.

To carry out such comparison a typical roughness of $0.046 \mathrm{~mm}$ was used as well as three pipe diameters 1", 15" and 40". As can be seen Panhandle B equation, although only dependent upon the Reynolds number closely follows AGA for medium and large diameters. It is also seen that Weymouth equation overestimates the friction coefficient when compared to AGA results and this difference increases with the pipe diameter reduction. As far as the Modified Colebrook-White friction factor evolution is concerned, this was calculated for two pipe diameters $1^{\prime \prime}$ and 40" and, as expected, this equation results are similar to those obtained with AGA equation for high Reynolds.

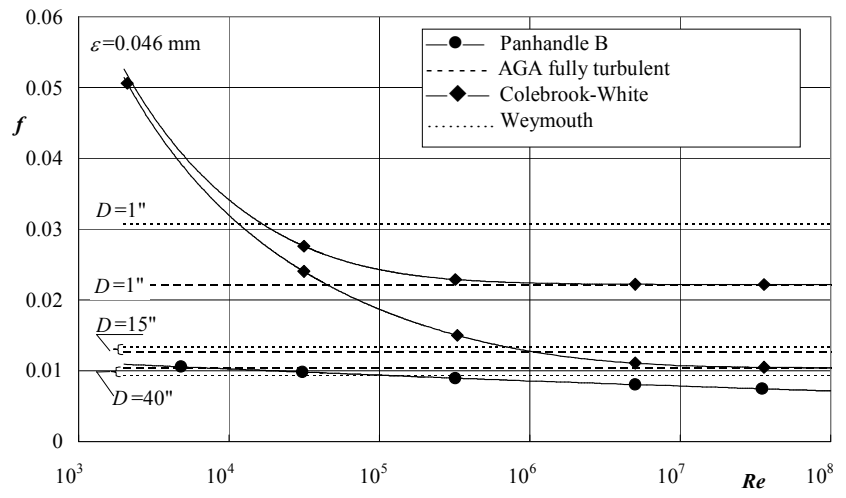

Figure 4 Friction coefficients used in the Panhandle B, AGA fully turbulent, Colebrook-White and Weymouth.

In most of the literature and for both equations, Panhandle A and $\mathrm{B}$, the exponent of the ratio $T_{s t} / P_{s t}$ is different from the unity contrary to the versions presented herein. Such difference is understandable if it is realized that in the present text a general form of Reynolds number equation has been used, Eq. (23), while in the developments appearing in the literature the Reynolds Eq. (24) was used. In this last equation the standard temperature and pressure were replaced by $288.15 \mathrm{~K}$ and $1 \mathrm{~atm}$, whereas such was not done to the same ratio, $T_{s t} / P_{s t}$, in the flow Eqs. (15) or (41). There is then a contradiction on the way the equation was developed and this results in the fact that the Panhandle A and B equations found in the some literature only give correct results if the standard temperature and pressure to be used with them are the same as those previously used in the definition of the Reynolds number, i.e., $288.15 \mathrm{~K}$ and $1 \mathrm{~atm}$.

\section{Other Equations for Steady State Flow}

\section{IGT Distribution Equation}

In this case the transmission factor is given by,

$$
\frac{1}{\sqrt{f}}=2.3095 \operatorname{Re}^{0.100}
$$

and using now Eq. (41) where $\alpha=2.3095$ and $\beta=0.100$, the following equation is obtained,

$$
\dot{Q}_{s t}=24.6145 \frac{1}{\mu^{1 / 9}} \frac{T_{s t}}{P_{s t}}\left[\frac{\left(P_{1}^{2}-P_{2}^{2}\right)-E}{L d^{8 / 10} T_{a v g} z_{s v g}}\right]^{5 / 9} D^{8 / 3}
$$

where the average compressibility factor $z_{\text {avg., }}$ is assumed as 1 .

\section{Mueller Equation}

For this case the transmission factor is given by,

$$
\frac{1}{\sqrt{f}}=1.675 \operatorname{Re}^{0.130}
$$

and following the same methodology as in the previous section, 


$$
\dot{Q}_{s t}=15.7650 \frac{1}{\mu^{0.1494}} \frac{T_{s t}}{P_{s t}}\left[\frac{\left(P_{1}^{2}-P_{2}^{2}\right)-E}{L d^{0.74} T_{\text {avg }} z_{\text {avg }}}\right]^{0.5747} D^{2.724}
$$
as 1 .

Once again the average compressibility factor $z_{\text {avg. }}$, is assumed

\section{Fritzsche Equation}

Now the transmission factor is given by,

$$
\frac{1}{\sqrt{f}}=3.3390(\operatorname{Re} D)^{0.071}
$$

and reworking Eq. (41),

$$
\dot{Q}_{s t}=39.2220 \frac{1}{\mu^{0.07643}} \frac{T_{s t}}{P_{s t}}\left[\frac{\left(P_{1}^{2}-P_{2}^{2}\right)-E}{L d^{0.858} T_{\text {avg }} z_{\text {avg }}}\right]^{0.5382} D^{2.6911}
$$

Using the approach of $\mu=1.0415 \times 10^{-5} \mathrm{~Pa} \mathrm{~s}$ and assuming that $z_{\text {avg, }}$ is equal to unity, the last equation becomes

$$
\dot{Q}_{s t}=94.2565 \frac{T_{s t}}{P_{s t}}\left[\frac{\left(P_{1}^{2}-P_{2}^{2}\right)-E}{L d^{0.858} T_{a v g}}\right]^{0.5382} D^{2.6911}
$$

\section{Pole Equation}

In this equation as well as in the next ones, and similarly to the Weymouth equation, the transmission factor is only dependent upon the pipe inside diameter. For the Pole equation the transmission factor will take the values shown in Table 2 (Mohitpour et al., 2000).

Table 2. $1 / \sqrt{f}$ for Pole equation.

\begin{tabular}{|c|l|}
\hline Pipe nominal diameter (inches) & $1 / \sqrt{f}$ \\
\hline $3 / 4$ to 1 & 4.78 \\
\hline $1 \frac{1}{4}$ to $1 / 2$ & 5.255 \\
\hline 2 & 5.735 \\
\hline 3 & 6.215 \\
\hline 4 & 6.45 \\
\hline
\end{tabular}

As the Pole equation is valid for low pressure flows, to obtain such equation the transmission factor according to what is presented in Table 2 is introduced in equation (39). In the technical literature this Pole equation is presented while neglecting the potential energy term E, the compressibility factor is assumed one, $T_{s t}=273.15 \mathrm{~K}$, $T_{\text {avg }}=277.8 \mathrm{~K}$ and $P_{s t}=1.015598 \times 10^{5} \mathrm{~Pa}$. In such conditions, Eq. (39) becomes,

$$
\dot{Q}_{s t}=C\left[\frac{\left(P_{1}-P_{2}\right) D^{5}}{L d}\right]^{1 / 2}
$$

where $C$ is given in Table 3 .

For $P_{\text {avg }}^{\prime}$, see equation (39), a value of $P_{\text {avg }}^{\prime}=P_{s t}+390[\mathrm{~Pa}]$ was adopted so that $C$ values were identical to those found in the technical literature Mohitpour et al. (2000) for the Pole equation.
Table 3.Constant $C$ in Pole equation

\begin{tabular}{|l|l|}
\hline Pipe nominal diameter (inches) & $C$ \\
\hline $3 / 4$ to 1 & 4.635 \\
\hline $1 \frac{1}{4}$ to $1 \frac{1}{2}$ & 5.096 \\
\hline 2 & 5.561 \\
\hline 3 & 6.027 \\
\hline 4 & 6.255 \\
\hline
\end{tabular}

\section{Spitzglass (Medium Pressure) Equation}

In this equation the transmission factor is given by,

$$
\frac{1}{\sqrt{f}}=\left(\frac{88.5}{1+0.09144 / D+1.1811 D}\right)^{0.500}
$$

where $D[\mathrm{~m}]$ is the pipe inside diameter. Replacing $f$ in Eq. (35) it gives,

$$
\dot{Q}_{s t}=125.1060 \frac{T_{s t}}{P_{s t}}\left[\frac{\left(P_{1}^{2}-P_{2}^{2}\right)-E}{L d T_{\text {avg }} z_{\text {avg }}(1+0.09144 / D+1.1811 D)}\right]^{1 / 2} D^{2.5}(66)
$$

The expression usually found in the literature is however more simplified as the potential energy is not taken into account, the compressibility factor is assumed equal to one, $T_{s t}=T_{a v g}=288.8 \mathrm{~K}$ and $P_{s t}=1.01325 \times 10^{5} \mathrm{~Pa}$, giving as result the following equation for medium pressure transportation,

$$
\dot{Q}_{s t}=0.02094\left[\frac{\left(P_{1}^{2}-P_{2}^{2}\right) D^{5}}{L d(1+0.09144 / D+1.1811 D)}\right]^{1 / 2}
$$

\section{Spitzglass (Low Pressure) Equation}

In this case Eq. (65) of the transmission factor is replaced into Eq. (39) for low pressure instead of Eq. (35) for higher pressure. Then,

$$
\dot{Q}_{s t}=125.1060 \frac{T_{s t}}{P_{s t}}\left[\frac{\left(P_{1}-P_{2}\right) 2 P_{\text {avg }}^{\prime}-E}{L d T_{\text {avg }} z_{\text {avg }}(1+0.09144 / D+1.1811 D)}\right]^{1 / 2} D^{2.5}
$$

Once again and similarly to what happened with Pole equation, the low pressure Spitzglass equation is usually presented in the technical literature in a simpler form because the potential energy is not taken into account, the compressibility factor is again assumed equal to one, also $T_{s t}=T_{\text {avg }}=288.15 \mathrm{~K}$ and $P_{s t}=1.01325 \times 10^{5} \mathrm{~Pa}$. Finally, $P_{\text {avg }}^{\prime}=P_{s t}+1210[\mathrm{~Pa}]$ and so by using all these approaches the Spizglass equation for low pressure flows becomes,

$$
\dot{Q}_{s t}=9.50\left[\frac{\left(P_{1}-P_{2}\right) D^{5}}{L d(1+0.09144 / D+1.1811 D)}\right]^{1 / 2}
$$

\section{Renouard (Medium Pressure) Equation}

Renouard equations are frequently used in Portugal and Spain (Brucart, 1987; Andrés et al., 1989; Becco, 1989) for the sizing of gas lines. In the present situation the transmission factor according to Brucart (1987) is given by,

$$
\frac{1}{\sqrt{f}}=2.4112 \mathrm{Re}^{0.09}
$$


and replacing it in Eq. (41) with $\alpha=2.4112$ and $\beta=0.09$ gives the following equation,

$$
\dot{Q}_{s t}=26.4437 \frac{1}{\mu^{0.0989}} \frac{T_{s t}}{P_{s t}}\left[\frac{\left(P_{1}^{2}-P_{2}^{2}\right)-E}{L d^{0.82} T_{\text {avg }} z_{\text {avg }}}\right]^{1 / 1.82} D^{4.82 / 1.82}
$$

This last equation is only valid in the cases when $\dot{Q}\left[\mathrm{~m}^{3} / \mathrm{h}\right] / D$ $[\mathrm{mm}]<150$ (Brucart, 1987), i.e. this means that $R e<4 \times 10^{6}$, according to Eq. (25). For higher Reynolds numbers the transmission factor should be calculated through,

$$
1 / \sqrt{f}=2.1822 \operatorname{Re}^{0.10}
$$

which gives $1 / \sqrt{f}$ values $9 \%$ higher than those obtained through equation (70), in other words, using the transmission factor such as defined by equation (70) results in an overestimation of the friction losses.

Renouard (1952) also suggests for interior piping, branches and rising columns $\left(\operatorname{Re}<10^{4}\right)$, the use of the following equation,

$$
f=0.21 \mathrm{Re}^{-0.2}
$$

resulting in friction factors higher than those obtained by means of equation (70).

Using an absolute viscosity of $\mu=1.0757 \times 10^{-5} \mathrm{~Pa} \mathrm{~s}$, neglecting the potential energy term and assuming $T_{s t}=T_{a v g}=288.15 \mathrm{~K}, P_{s t}=$ $1.01325 \times 10^{5} \mathrm{~Pa}$ and that $z_{\text {avg }}=1$, Eq. (71) is reworked to give an equation applicable to medium pressure flows,

$$
\dot{Q}_{s t}=0.010367\left[\frac{\left(P_{1}^{2}-P_{2}^{2}\right)}{L d^{0.82}}\right]^{1 / 1.82} D^{4.82 / 1.82}
$$

In the technical literature the Renouard equation comes written in an explicit form in terms of the pressure difference and in such circumstances Eq. (74) becomes,

$$
P_{1}^{2}-P_{2}^{2}=4088 \dot{Q}_{s t}^{1.82} L d^{0.82} D^{-4.82}
$$

whereas in the technical literature (Brucart, 1987) it appears as,

$$
P_{1}^{2}-P_{2}^{2}=4810 \dot{Q}_{s t}^{1.82} L d D^{-4.82}
$$

where the constant 4810 takes the value 48600 if the following units are used, $P-\mathrm{kg} / \mathrm{cm}^{2}, L-\mathrm{km}, \dot{Q}-\mathrm{m}^{3} / \mathrm{h}$ and $D-\mathrm{mm}$.

It must be stressed that in the development leading to equation (76), Renouard (1952) used a constant value for city gas kinematic velocity, $v_{c g}=2.2 \times 10^{-5} \mathrm{~m}^{2} / \mathrm{s}$, and a flow temperature of $15^{\circ} \mathrm{C}$. This means that by fixing the value of gas cinematic viscosity, the density is also kept fixed, which is physically inaccurate when considering compressible gas flows at medium or high pressure, because the cinematic viscosity of gases is highly dependent upon pressure. The gas flow Reynolds number is now defined as

$$
\operatorname{Re}=\frac{4 \dot{Q}_{s t}}{\pi D 2.2 \times 10^{-5}}
$$

and every time a gas with a cinematic viscosity $v$ different from the city gas is being used, a multiplying correction factor $\left(v / v_{c g}\right)^{0.18}$ must be applied into Eq. (76), Renouard (1952). The product between such correction factor and the relative density of gas $d\left(v / v_{c g}\right)^{0.18}$ is usually called the corrected density.

To get a deeper understanding of the reasoning and mathematical simplifications carried out by Renouard (1952), it is necessary to go back and rework Eq. (22) for the gas flow Reynolds number. Such equation can thus be written as

$$
\operatorname{Re}=\frac{4 \dot{Q}_{s t}}{\pi D\left(\frac{v \rho}{\rho_{s t}}\right)}
$$

which means that this equation is identical to Eq. (77) whenever the used viscosity is an apparent viscosity calculated through

$$
v_{a p p}=\frac{v \rho}{\rho_{s t}}=\frac{\mu}{\rho_{s t}} \cong v_{s t}
$$

The correction factor to be applied into Eq. (76) when a gas different from the city gas originally used by Renouard (1952) is being used, or because a different temperature is being considered, must then be calculated through one of the following ratios

$$
\left(\frac{\mu / \rho_{s t}}{2.2 \times 10^{-5}}\right)^{0.18}=\left[\frac{\mu /\left(d \rho_{s t, a i r}\right)}{2.2 \times 10^{-5}}\right]^{0.18}=\left[\frac{\mu /(d 1.26)}{2.2 \times 10^{-5}}\right]^{0.18}
$$

Comparing Eqs. (75) and (76) and considering what has been previously exposed, the error obtained in the calculation of the pressure differential by using Eq. (76) instead of Eq. (75) is given by

$$
\left[\left(P_{1}^{2}-P_{2}^{2}\right)_{\mathrm{Eq} \cdot(76)}-\left(P_{1}^{1}-P_{2}^{2}\right)_{\mathrm{Eq} \cdot(75)}\right] /\left(P_{1}^{2}-P_{2}^{2}\right)_{\mathrm{Eq} \cdot(75)}
$$

and so if in Eq.(75) it is assumed that the constant, 4088, changes according to the gas viscosity and in Eq. (76) a corrected gas density is used according to Eq. (80), the Eq. (81) becomes,

$$
\frac{4810 d\left(\mu / \rho_{s t}\right)^{0.18} /\left(2.2 \times 10^{-5}\right)^{0.18}}{4088 \mu^{0.18} d^{0.82} /\left(1.0757 \times 10^{-5}\right)^{0.18}}-1
$$

leading after simplification to the following result,

$$
\frac{4810}{4088}\left(\frac{1.0757}{2.2}\right)^{0.18}\left(\frac{1}{\rho_{\text {st }, \text { air }}}\right)^{0.18}-1=-0.8 \%
$$

which means that both Eqs. (75) and (76) are equivalent. However, this result suggest that Eq.(75) should be used instead of Eq. (76), because that equation was not only deduced through a physically correct way, but it does not require the use of the corrected density, provide in Eq. (71) the typical density for the gas being used is applied. For an even higher correctness it would be necessary to use a viscosity calculated for average flow conditions.

The use of Eq.(76) without the viscosity correction, although quite common in the daily practice, leads to an overestimation on the calculation of the pressure drop, as the pressure difference $P_{1}^{2}-P_{2}{ }^{2}$ is about 6 to $9 \%$ higher than the value obtained through Eq. (75). 


\section{Renouard (Low Pressure) Equation}

For low pressure, Eq. (70) is still being used for the transmission factor but the pressure drop equation is based upon Eq. (39). It is then obtained that,

$$
\dot{Q}_{s t}=26.4437 \frac{1}{\mu^{0.0989}} \frac{T_{s t}}{P_{s t}}\left[\frac{\left(P_{1}-P_{2}\right) 2 P_{\text {avg }}^{\prime}-E}{L d^{0.82} T_{\text {avg }} z_{\text {avg }}}\right]^{1 / 1.82} D^{4.82 / 1.82}
$$

As in the previous situation, assuming that $\mu=1.0757 \times 10^{-5} \mathrm{~Pa} \mathrm{~s}$, ignoring the potential energy term and considering that $T_{s t}=T_{\text {avg }}=$ $288.15 \mathrm{~K}, P_{s t}=1.01325 \times 10^{5} \mathrm{~Pa}$ and $z_{\text {avg }}=1$ a simpler equation can be obtained

$$
\dot{Q}_{s t}=8.614\left[\frac{\left(P_{1}-P_{2}\right)}{L d^{0.82}}\right]^{1 / 1.82} D^{4.82 / 1.82}
$$

The $P_{a v g}^{\prime}$ is defined as $P_{a v g}^{\prime}=P_{s t}+1390[\mathrm{~Pa}]$ (This pressure value is used to convert from high to low pressure equations found in the literature). Such mathematical treatment is equivalent to replace $P_{1}^{2}-P_{2}^{2}$ by $\left(P_{1}-P_{2}\right) 2 P_{\text {avg }}^{\prime}$ in Eqs. (74) to (76). The efficiency factor used with Renouard equations is usually 0.91 which is equivalent to use, in the flow equation, an equivalent length of $1.2 \mathrm{~L}$.

\section{Laminar Flow Regime}

The laminar flow can occur in domestic gas pipes which justifies the deduction of the flow equation for this situation.

The transmission factor is given by,

$$
\frac{1}{\sqrt{f}}=K \operatorname{Re}^{1 / 2}
$$

where $K$ is equal to $1 / \sqrt{f_{a p p} \operatorname{Re}}$ where the value of $f_{a p p}$ Re is given by Eq. (28). Replacing the definition of the transmission factor for laminar flow into Eq. (41) and solving it in order to $\dot{Q}_{s t}$,

$$
\dot{Q}_{s t}=\frac{\pi}{4} \frac{1}{\left(f_{\text {app }} \mathrm{Re}\right)} \frac{1}{\mu} \frac{T_{s t}}{P_{s t}}\left[\frac{\left(P_{1}^{2}-P_{2}^{2}\right)-E}{L T_{\text {avg }} z_{\text {avg }}}\right] D^{4}
$$

Neglecting the potential energy term $E$, assuming that $T_{\text {avg }}=T_{s t}$ $=288.15 \mathrm{~K}, z_{\text {avg }}=1$ and $\mu=1.0757 \times 10^{-5} \mathrm{~Pa} \mathrm{~s}$ and adapting the last equation exclusively for low pressure flow calculations by doing $P_{\text {avg }}^{\prime}=P_{s t}+390[\mathrm{~Pa}]$, the result is,

$$
\dot{Q}_{s t}=\frac{146588}{\left(f_{a p p} \operatorname{Re}\right)} \frac{\left(P_{1}-P_{2}\right)}{L} D^{4}
$$

which is the simplified flow equation for laminar, low pressure regime.

Figure 5 presents a comparison among several friction factors for partially and fully turbulent flow regimes. In the situations when the pipe diameter and roughness are essential for the calculation of $f$, it was assumed for calculation purposes that $D=4$ " and steel pipe with $\varepsilon=0.046 \mathrm{~mm}$ roughness (White, 1999).

From the analysis of Fig. 5 it is clear that the Muller equation gives $f$ values approximated to those of AGA partially turbulent equation (AGA p/t), for Reynolds numbers up to $4 \times 10^{4}$, whereas the
IGT equation gives values quite close to those of AGA $\mathrm{p} / \mathrm{t}$ precisely beyond $\mathrm{Re}=4 \times 10^{4}$. The combination of Muller and IGT equations is a good alternative to the use of AGA $p / t$ equation as those two are explicit equations while AGA $p / t$ equation is an implicit one, thus more difficult to deal with. Still keeping an eye on Fig. 5 it is observed that the friction coefficients from Renouard equations are inferior to those obtained from AGA $\mathrm{p} / \mathrm{t}$ for Reynold numbers smaller than $7.4 \times 10^{3}$, which means that the value of $f$ is underestimated when using the Renouard equation. However, for Reynolds numbers above $7.4 \times 10^{3}$ the friction factor for the Renouard equation is superior to the value obtained with AGA $p / t$ equation; in this case there is an overestimation of the friction factor which places the designer on the safe side during equipment and piping design. The Fristzsche equation has an overall behavior quite similar to the Renouard equation, although giving higher friction factors when $\operatorname{Re}>7.4 \times 10^{3}$.

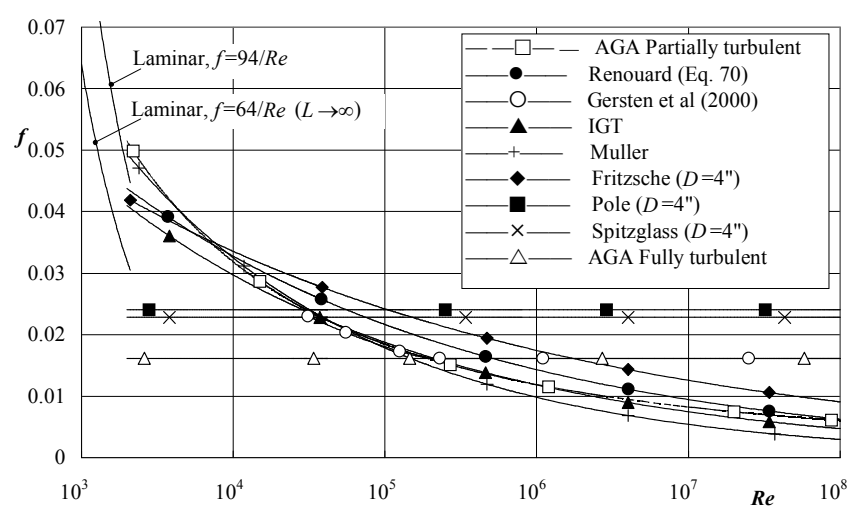

Figure 5 Evolution of friction factors used in several, previously discussed, flow equations.

Equations of Pole and Spitzglass, because they are only function of the pipe diameter should, in theory, be most adequate for fully turbulent flow where they seem to overestimate the value of $f$ when compared with the fully turbulent AGA equation. In the partially turbulent flow, for low Reynolds numbers and small pipe diameters, Pole and Spitzglass equations still can go on overestimating the friction factor as this factor greatly increases with the decrease of pipe internal diameter. In fact, for $D=3 / 4 " f$ is equal to 0.0438 or 0.06579 according to Pole or Spitzglass equations, however for large pipe diameters and small Reynolds these equations may underestimate the value of $f$ as it is clearly seen in Fig. 5. Once again is clear that the physical support of the equations is weak and consequently their used should be avoided whenever possible.

The use of turbulent flow equations for laminar flow situations will impose an overestimation of the friction coefficient, although in the majority of practical situations the minimum Reynolds number is 1400. As can be seen in Fig. 5 for a long duct, when the entrance length effect can be neglected and consequently $f_{a p p} \mathrm{Re}=64$, the friction factor obtained through equations of Renouard, Fritzsche and IGT is inferior to the one obtained through adequate correlations for laminar flow, but only for Reynolds smaller than 1400. The same happens for AGA $\mathrm{p} / \mathrm{t}$ and Muller equations when the Reynolds number is inferior to 1000 . However, for shorter pipes, a common situation in buildings and small gas networks, the laminar friction coefficient is higher than those obtained by the turbulent equations. For a duct with $80 \mathrm{D}$ length the product $f_{a p p}$ Re is close to 94 and according to what is observed in Fig. 5, in this situation the laminar friction factor is always superior to the value obtained by means of any other turbulent equations. However, practical minimum diameters in domestic installations are well above the 
sizes suggested by these laminar calculations and accordingly this analysis is purely academic.

Figure 6 presents the maximum variation detected for the friction factor in relation to the average value calculated by means of the AGA partially turbulent, Renouard, Muller, IGT and laminar flow equations as a function of the Reynolds number. There is a minimum dispersion around $5 \%$ for Reynolds closer to $1.6 \times 10^{4}$, but such dispersion increases for extreme Reynolds number, be they very small or very large.

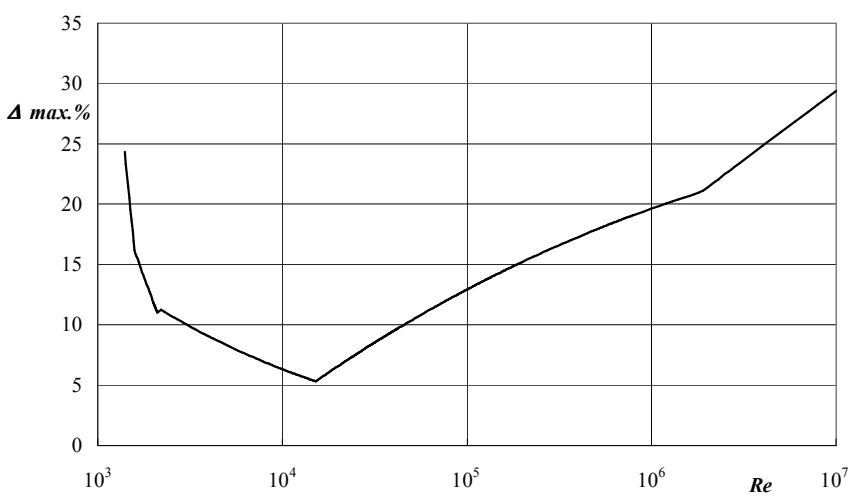

Figure 6 Maximum variation of the friction factor relatively to its average value when calculated through the following equations: AGA p/t, Renouard, Muller, IGT and for laminar flow regime.

From this analysis it can be said that in the calculation and design for distribution and or utilization gas networks in partially turbulent regime, which is nowadays highly dependent upon numerical procedures, such calculation procedure could be carried out through the following explicit equations (for pressure calculations): for Reynolds above 2100, White Eq. (47), although this equation will still be implicit in terms of $D$ and $\dot{Q}$, while in the laminar regime $\operatorname{Re}<2100$, Eq. (88) is the recommended correct option, although for practical reasons it is not frequent the use of very small $(<18 \mathrm{~mm})$ pipe diameters, making the use of this last equation not very common. In all these recommended equations the average flow temperature is dependent upon the designer choice and the gas absolute viscosity is calculated in accordance with such temperature.

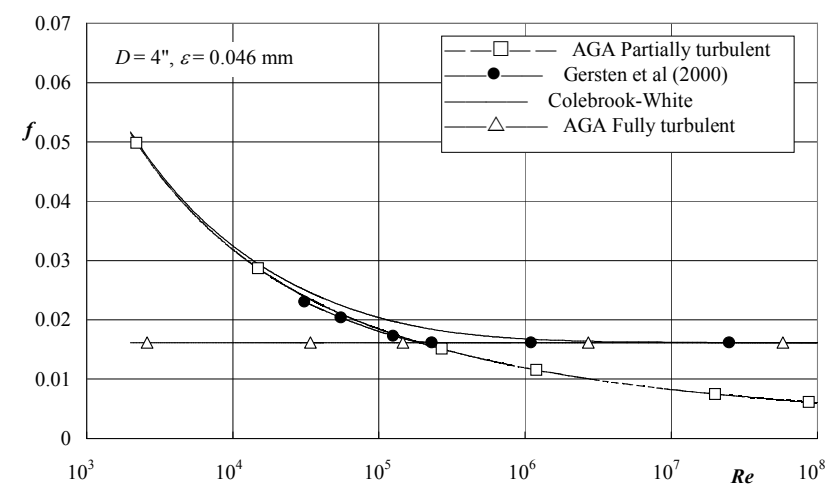

Figure 7 Comparison among AGA, Colebrook-White and Gersten et al equations (2000).

Figure 7 presents the performance of several flow equations at the transition between partially and fully turbulent flow regimes for a 4 " pipe with $0.046 \mathrm{~mm}$ of absolute roughness. The best equation to translate the experimental behavior is the Gersten et al. (2000) with the abrupt transition between the two flow regimes. Both AGA equations, using Eq. (32) as selection criterion, closely follow the Gersten et al. (2000) equation (and accordingly the experimental data) and at the same time are formally simpler to use. In this Fig. 7 it is also evident the inadequacy of the modified Colebrook-White equation to translate correctly the experimental behavior in the transition region.

\section{Conclusions}

In spite of the large amount of work carried out on natural gas flow it is quite useful to join in a single text the multiplicity of equations that are dispersed in the vast amount of the available technical literature. It is then shown that the basic difference among the several presented and discussed equations, is not in their formal aspect, but lies in the corresponding transmission coefficient equations. There is however a unique equation in this universe that differs from all the others, not only by means of the transmission coefficient equation, but also through the way the flow equation is deduced, the Renouard equation.

The comparison among the different flow equations using AGA equations as a reference basis was fundamental to the definition, for each equation under analysis, of the corresponding application range and errors arriving from their use. It is, as far as the authors are concerned, the first time all these equations are presented together in S.I. units. The main conclusions of this work are:

i) It is possible, through a very simple equation, to correlate the relative pipe roughness $\varepsilon / D$ with the critical Reynolds corresponding to the transition from partially to fully turbulent natural gas flow;

ii) According to practical limitations for natural gas pipeline flow (Kennedy, 1993) and taking into account typical pipe roughness, it is possible to conclude that for pressures below 5 bar and for cooper and polyethylene pipes, the only available flow regime is the partially turbulent flow and in such circumstances the pipe roughness is not required for calculation purposes. However, for steel pipes the existence of fully turbulent flow is common and it is not correct to use in the calculations flow equations that ignore the influence of internal pipe roughness;

iii) Low pressure flow equations are simplifications of the flow equation which was obtained through the integration of the equation of linear momentum conservation for compressible fluids. The result is an equation of limited scope, as it can only be applied to a limited pressure range, i.e. $<50$ mbar;

iv) The use of a formula for the calculation of the Reynolds number where $T_{\text {st }}$ e $P_{\text {st }}$ are previously replaced by the corresponding numerical values, imposes that on the deduction of the flow equations the exponent of the ratio $\left(T_{\mathrm{st}} / P_{\mathrm{st}}\right)$ is different from unity. On the other end, if during calculations carried out with this flow equation the values used for $T_{\mathrm{st}}$ e $P_{\mathrm{st}}$ are different from those used in the calculation of Re, there will be an error in the numerical results;

v) The utilization of the AGA equations, together with the equation for the determination of $\mathrm{Re}_{\mathrm{cr}}$ recommended in the present text, is a good alternative to the Gersten et al. (2000) equation, because AGA equations are formally simpler and translate equally well the abrupt change found in the transition from partially to fully turbulent flow of natural gas.

vi) Equation (75) should be used instead of Eq. (76) when calculating natural gas pipelines through the equation of Renouard.

\section{References}

Andrés, J.A., Pomatta R., Lastra S.A. and Gándara M.G. 1997, "Gaseous Fuels Installations" (In Spanish), Ediciones A. Madrid Vicente, Madrid. 
Becco, J.L.L. 1989, “Liquified Petroleum Gases” (In Spanish), RepsolButano, S.A., Madrid.

Branco, J.F., Pinho, C. and Figueiredo, R.A. 2001, "From a power-law equation for the friction factor in smooth pipes to a controversy on the overlap layer", Proceedings of COBEM 2001, Fluid Mechanics, Vol. 8, pp 21-29.

Brucart, E.B. 1987, "Natural Gás” (In Spanish), Editores Técnicos Asociados, S.A., Barcelona.

Gersten, K., Papenfuss, H.H., Kurschat, T., Genillon, P., Fernández Pérez, F. and Revell, N., 2000, "New Transmission-factor formula proposed for gas pipelines", Oil \& Gas Journal, Feb. 14, pp.58-62.

Katz, D.L. and Lee, R.L., 1990, "Natural Gas Engineering: Production and Storage", McGraw-Hill, New York.

Kennedy, J.L., 1993, "Oil and Gas Pipeline Fundamentals”, $2^{\text {nd }}$ edition, Pennwell Books.

Mohitpour, M., Golshan, H. and Murray, A., 2000, "Pipeline design \& construction: A practical approach", ASME Press.

Munson, B.R., Young, D.F. and Okiishi, T.H., 1998, "Fundamentals of fluid mechanics", John Wiley \& Sons.

Osiadacz, A.J., 1987, "Simulation and analysis of gas networks", E. \& F.N. SPON, London.
Pritchard, R., Guy, J.J. and Connor, N.E, 1978, "Industrial Gas Utilization, Engineering Principles and Practice", Bowker Publishing Company, Epping, Essex

Renouard, M.P., 1952, Nouvelles règles à calcul pour la détermination des pertes de charge dans les conduites de gaz, Journal des Usines à Gaz, Octobre, pp 337-339.

Schroeder, D. W. 2001, "A tutorial on pipe flow equations", www.psig.org.

Shah, R.K. and London, A. L., 1978, "Laminar flow forced convection in ducts", Academic Press.

Smith, R.V., Miller, J.S. and Fergus, J.W., 1956, "Flow of Natural Gas Through Experimental Pipe Lines and Transmission Lines", Monograph 9, US Department of Interior, Bureau of Mines, Publisher: American Gas Association, New York.

Smith, R.V., 1990, "Practical Natural Gas Engineering", PennWell Books.

White, F.M., 1979, "Fluid Mechanics", $1^{\text {st }}$ edition, International Student Edition, McGraw-Hill, New York.

White, F.M., 1999, "Fluid Mechanics", $4^{\text {th }}$ edition, McGraw-Hill, New York. 